\title{
Atendimento ambulatorial de pacientes com diabetes mellitus tipo 2
}

\author{
Outpatient care of patients with type 2 diabetes mellitus
}

Atención ambulatoria de pacientes con diabetes mellitus tipo 2

Daniela Buriol $^{1 \star}$, Laura Hossa Palmeiro ${ }^{1}$, Gisele Miollo ${ }^{1}$, Maria Denise Schimith ${ }^{1}$, Dalva Cezar da Silva ${ }^{1}$, Luiza Camila Jerke'1.

\section{RESUMO}

Objetivo: Comparar as características sociodemográficas, clínicas e os cuidados com os pés dos pacientes com Diabetes Mellitus tipo 2 atendidos na unidade ambulatorial de um Hospital Universitário. Métodos: Pesquisa quantitativa e comparativa entre amostras de 106 e 85 pacientes com Diabetes Mellitus tipo 2, atendidos pela consulta médica e de enfermagem respectivamente. Coletou-se os dados por meio de formulário. A primeira amostra no período de julho a agosto de 2016 e a segunda nos períodos de agosto a julho de 2018 e maio a julho de 2019. Realizou-se análise estatística descritiva e analítica. Resultados: Predominância do sexo feminino $(68,9 \%)$ na primeira amostra e do sexo masculino $(68,24 \%)$ na segunda. Prevalência de amputação prévia no sexo masculino, com $60 \%$ e $71,69 \%$, respectivamente e renda igual ou inferior a um salário mínimo nas amostras. Os pacientes foram orientados por profissionais da saúde. Ademais, encontrou-se divergências quanto ao tipo de calçado utilizado e a presença de alterações nas unhas entre as amostras. Conclusão: $O$ conhecimento das informações desse estudo contribuirá à atuação da enfermagem e desenvolvimento de educação em saúde.

Palavras-chave: Assistência ambulatorial, Diabetes mellitus tipo 2, Enfermagem, Promoção da saúde.

\begin{abstract}
Objective: To comparing the sociodemographic, clinical characteristics and foot self-care at patients with Diabetes Mellitus type 2 attended by an ambulatory unit of the University Hospital. Methods: Comparative and quantitative research between two samples of 106 and 85 patients with type 2 Diabetes Mellitus attended by medical and nursing consultation, respectively. We collected the data through a formulary. The first sample was collected in the period between July to August in 2016. The second one was collected in the periods between August to December in 2018 and May to July in 2019. We submitted the data to descriptive critical analysis. Results: Most of the patients were female in the first sample $(68,9 \%)$, and male $(68,24 \%)$ in the second one. The previous amputation was predominant in male sex, with $60 \%$ and $71,69 \%$, respectively and minimum wage income in both samples. Health professionals counseled the patients. Additionally, in both samples, we found deviations about the type of footwear and nail changes. Conclusion: The study provides us valuable information for nursing performance evaluation and health education development.
\end{abstract}

Keywords: Ambulatory care, Diabetes mellitus type 2, Nursing, Health promotion.

\section{RESUMEN}

Objetivo: Comparar las características sociodemográficas, clínicas y el autocuidado de los pies de pacientes con Diabetes Mellitus tipo 2 atendidos en una unidad ambulatoria de un hospital universitario. Métodos: Investigación cuantitativa y comparativa entre muestras de 106 y 85 pacientes con Diabetes Mellitus tipo 2 atendidos por la consulta médica y por la enfermería. Los datos fueron recogidos por medio de una forma. La

${ }^{1}$ Universidade Federal de Santa Maria (UFSM), Santa Maria - RS. *E-mail: burioldani@hotmail.com 
primera muestra fue recogida en el período de julio a agosto en el año de 2016 y la segunda muestra en el período de agosto a julio en 2018 y de mayo a julio en 2019. Se realizó análisis estadístico descriptivo y analítico. Resultados: En la primera muestra predominaron las mujeres $(68,9 \%$ y en la segunda muestra los hombres (68,24\%). Es de señalar el predominio de amputación previa en los hombres, con $60 \%$ y $71,69 \%$, respectivamente y ingresos menores 0 iguales a un salario mínimo. Los pacientes fueron guiados por profesionales de la salud. En ambas muestras fueron encontradas diferencias entre los tipos de zapato y cambios en las uñas. Conclusión: El conocimiento de la información del estudio ha contribuido con la evaluación en enfermería y en desarrollo de prácticas en educación para la salud.

Palabras clave: Atención ambulatoria, Diabetes mellitus tipo 2, Enfermería, Promoción de la salud.

\section{INTRODUÇÃO}

O Diabetes Mellitus (DM) é uma das Doenças Crônicas Não Transmissíveis (DCNT) que está entre as dez principais causas de morte do mundo, com quase metade ocorrendo em pessoas com menos de 60 anos. Por esse motivo, configura-se um importante e crescente problema global de saúde do século XXI. Em 2019, estimou-se que cerca de 1 em 11 adultos entre 20 e 79 anos possuíam a doença, o que equivale a uma prevalência de $9,3 \%$ correspondente a 463 milhões de pessoas. Se essas tendências continuarem, em 2030, serão 578 milhões de casos, e em 2045, cerca de 700 milhões de pessoas estarão vivendo com DM. Avaliase que $79,4 \%$ dos adultos com a doença estão localizados em países de baixa e média renda (INTERNATIONAL DIABETES FEDERATION, 2019).

O DM tipo 2 é um distúrbio metabólico presente em 90 a 95\% das pessoas acometidas por diabetes no mundo todo. Possui uma etiologia complexa envolvendo fatores de origem genética e ambiental. Trata-se de uma doença poligênica, com forte herança familiar. Seus principais fatores de risco são: inatividade física, dieta inapropriada, obesidade, diagnóstico prévio de pré-diabetes ou diabetes mellitus gestacional, presença de hipertensão e dislipidemia (SOCIEDADE BRASILEIRA DE DIABETES, 2019a). Geralmente, os idosos são os mais acometidos pela doença. Contudo, em alguns países o aumento da incidência do DM tipo 2 foi observado em crianças e jovens devido a presença de fatores de risco associados a comportamentos nocivos ao organismo como o hábito de fumar e o consumo de álcool (INTERNATIONAL DIABETES FEDERATION, 2019; SOCIEDADE BRASILEIRA DE DIABETES, 2019a).

O DM tipo 2 possui diversas complicações crônicas, sendo o pé diabético a mais comum e agressiva. A prevalência de úlceras nos pés atinge de $4 \%$ a $10 \%$ das pessoas com a doença e geralmente evolui para amputações não traumáticas de membros inferiores. O cuidado com essas lesões exige grande responsabilidade, tanto do profissional de saúde quanto do paciente e sua família. Já que, devido a sua condição clínica necessita manter o controle glicêmico, além de adaptar-se às limitações que as ulcerações e amputações trazem para sua vida. Elas podem dificultar a realização de afazeres diários e até mesmo exigir um afastamento do trabalho, podendo culminar no surgimento de outras comorbidades, como a depressão, por exemplo (SOCIEDADE BRASILEIRA DE DIABETES, 2019a; INTERNATIONAL WORKING GROUP ON THE DIABETIC FOOT, 2019).

No mundo, o DM configura-se como uma epidemia silenciosa, cuja taxa de crescimento ao longo dos anos vem aumentando acentuadamente, gerando ainda mais aumento nos custos de saúde (INTERNATIONAL DIABETES FEDERATION, 2019; SOCIEDADE BRASILEIRA DE DIABETES, 2019a). Por esse motivo, ressalta-se a necessidade de identificar os elementos envolvidos nessa doença, como os determinantes sociais, os grupos populacionais vulneráveis e as faixas etárias. Ademais, é importante identificar a evolução do $\mathrm{DM}$, suas complicações, taxas de morbimortalidade e fatores de risco, devido à relevância da problemática no país e no mundo (BRASIL, 2015).

Estudos existentes demonstram, em relação aos dados sociodemográficos, prevalência de pessoas do sexo feminino, baixo nível de escolaridade, idosos, além da presença de hipertensão arterial sistêmica como principal comorbidade associada (NETO MO, et al., 2017; SILVA JMTS, et al., 2017; TESTON EF, et al., 2017). Quanto aos cuidados com os pés, destaca-se a presença de práticas adequadas como não caminhar 
descalço e, de condutas ou condições inadequadas como a não utilização de calçados específicos para diabéticos e a presença de calosidades (NETO MO, et al., 2017; SILVA JMTS, et al., 2017; TESTON E, et al., 2017; CARLESSO GP, et al., 2017).

Cabe ainda destacar que a partir de pesquisas sobre o DM pode-se realizar o reconhecimento das características clínicas e socioeconômicas das pessoas com DM tipo 2, que poderá gerar informações importantes para contribuir no desenvolvimento e avaliação de diretrizes, políticas, programas e serviços de saúde. Além de colaborar para o desenvolvimento de novos métodos diagnósticos e terapêuticos. Assim sendo, é necessário que os enfermeiros tenham pleno conhecimento destes elementos para poderem auxiliar no planejamento de ações de saúde, desenvolver planos de cuidados de enfermagem e prestar as devidas orientações sobre DM e pé diabético. Desse modo, esses profissionais são essenciais e de grande importância para a promoção da saúde, prevenção, diagnóstico e tratamento de DM tipo 2 (BRASIL, 2015).

Dessa forma, estabeleceu-se a seguinte questão norteadora: há diferença nas características sociodemográficas e clínicas, e os cuidados com os pés realizados pelos pacientes com DM tipo 2 que estavam em acompanhamento com a unidade ambulatorial de um Hospital Universitário (HU) durante o período de 2016, 2018 e 2019? Assim, o presente estudo objetiva comparar as características sociodemográficas, clínicas e os cuidados com os pés dos pacientes com DM tipo 2 atendidos em uma unidade ambulatorial de um Hospital Universitário.

\section{MÉTODOS}

Trata-se de uma pesquisa quantitativa, descritiva e comparativa entre duas amostras. A primeira contendo 106 pacientes com DM tipo 2 oriundos da consulta médica. Foi desenvolvida no período de julho a agosto de 2016. A segunda amostra foi de 85 pacientes com DM tipo 2 que estavam em acompanhamento com a equipe de enfermagem, nos períodos de agosto a dezembro de 2018 e de maio a julho de 2019. A pesquisa foi realizada em uma unidade ambulatorial de um HU do Sul do Brasil com a amostra final de 191 pacientes entrevistados.

Os participantes da pesquisa foram pacientes adultos de ambos os sexos. Os critérios de inclusão foram: ter idade igual ou superior a 18 anos, possuir diagnóstico de DM tipo 2 e estar em acompanhamento no Ambulatório do Hospital Universitário pesquisado. Para o cálculo do tamanho amostral foi considerada uma população total de 628 pacientes com DM tipo 2 atendidos no período de setembro de 2014 a setembro de 2015. Utilizou-se um grau de confiança de 1,645 , que corresponde a um nível de confiança de $90 \%$, e erro amostral de 0,05.

O formulário, construído especificamente para a pesquisa, foi aplicado em forma de entrevista semiestruturada e preenchido pelos pesquisadores, para realizar a caracterização dos pacientes, contendo questões como: número do registro, sexo, idade, situação econômica e grau de instrução. Quanto aos aspectos clínicos foram investigados: os fatores de risco, amputação prévia, a presença de dor em repouso, edema, formigamento, dormência, se era tabagista, etilista e se realizava atividade física.

Também foram investigados os cuidados com os pés nas seguintes questões: uso de hidratante, hábito de andar descalço, tipo de calçado, uso de meias em calçados fechados, corte das unhas, presença de rachaduras, calos, alterações nas unhas, e se recebeu orientação de algum profissional. Ressalta-se que após a entrevista, os pacientes eram orientados quanto às práticas de cuidado conforme as necessidades e dúvidas.

Anteriormente às entrevistas, ocorreu o contato com as equipes do ambulatório para explicar sobre o procedimento da coleta de dados. Os participantes foram informados sobre todos os aspectos da pesquisa, e caso houvesse interesse em participar, o Termo de Consentimento Livre e Esclarecido era fornecido em duas vias para leitura e assinatura. Uma via ficava na posse do participante e a outra permanecia na posse do pesquisador. As coletas dos dados foram realizadas por estudantes de graduação e pós-graduação em enfermagem previamente capacitados.

Os dados coletados foram duplamente digitados por dois pesquisadores, havendo a verificação de erros e inconsistências, para um controle de qualidade, no software Microsoft Excel versão 2016. Posteriormente, 
esses dados foram analisados por meio de estatística descritiva e analítica. Todas as análises foram realizadas por meio do software Statistical Package for the Social Sciences (SPSS) versão 17. Primeiramente, foi realizada a análise descritiva simples de todas as variáveis coletadas com frequências absolutas (n) e relativas (\%). Após, na analítica, foram realizadas análises de comparação entre os grupos pelo teste Quiquadrado (Qui 2). O nível de significância adotado foi de 0,05 para todos os testes.

A pesquisa foi desenvolvida de acordo com as considerações éticas exigidas pelas legislações que regulamentam a pesquisa com seres humanos no Brasil (BRASIL, 2012). O estudo foi aprovado pela Direção de Ensino e Pesquisa do HU e pelo Comitê de Ética e Pesquisa da UFSM em 07/06/2016, parecer número 1.578 .528 .

\section{RESULTADOS}

Dos 191 participantes entrevistados, 106 pertenciam à amostra de 2016 e 85 à amostra de 2018/2019. Observou-se que na primeira amostra havia mais pacientes do sexo feminino, sendo $73(68,9 \%)$, enquanto que na segunda amostra ocorreu o predomínio de pacientes do sexo masculino, com 58 (68,2\%). A seguir apresenta-se o perfil sociodemográfico em relação a sexo, faixa etária, renda e escolaridade (Tabela 1).

Tabela 1 - Características sociodemográficas dos pacientes em acompanhamento ambulatorial.

\begin{tabular}{|c|c|c|c|c|c|}
\hline \multirow{2}{*}{ Variáveis } & \multicolumn{2}{|c|}{2016} & \multicolumn{2}{|c|}{ 2018/2019 } & \multirow{2}{*}{$\mathbf{p}^{*}$} \\
\hline & $\mathbf{n}$ & $\%$ & $\mathbf{n}$ & $\%$ & \\
\hline \multicolumn{6}{|l|}{ Sexo } \\
\hline Masculino & 33 & 31,1 & 58 & 68,2 & \multirow{2}{*}{0,001} \\
\hline Feminino & 73 & 68,9 & 27 & 31,8 & \\
\hline \multicolumn{6}{|l|}{ Faixa Etária (em anos) } \\
\hline $30-39$ & 9 & 8,5 & 1 & 1,2 & \multirow{7}{*}{0,063} \\
\hline $40-49$ & 22 & 20,8 & 8 & 9,4 & \\
\hline $50-59$ & 20 & 18,9 & 21 & 24,7 & \\
\hline $60-69$ & 29 & 27,4 & 30 & 35,3 & \\
\hline $70-79$ & 20 & 18,9 & 21 & 24,7 & \\
\hline $80-89$ & 5 & 4,7 & 4 & 4,7 & \\
\hline $90-99$ & 1 & 0,9 & 0 & 0 & \\
\hline \multicolumn{6}{|c|}{ Renda (salário mínimo) ${ }^{\star \star}$} \\
\hline Zero a um & 47 & 45,2 & 47 & 55,3 & \multirow{4}{*}{0,415} \\
\hline Mais de um até dois & 29 & 27,9 & 22 & 25,9 & \\
\hline Mais de dois até três & 18 & 17,3 & 12 & 14,1 & \\
\hline Mais de três & 10 & 9,6 & 4 & 4,7 & \\
\hline \multicolumn{6}{|l|}{ Escolaridade } \\
\hline Nunca estudou & 5 & 4,7 & 9 & 10,6 & \multirow{4}{*}{0,212} \\
\hline 1 a 8 anos & 69 & 65,1 & 57 & 67,1 & \\
\hline 9 a 11 anos & 27 & 25,5 & 18 & 21,2 & \\
\hline 12 anos ou mais & 5 & 4,7 & 1 & 1,2 & \\
\hline
\end{tabular}

Legenda: $\left(^{*}\right)$ nível de significância $p \leq 0,05 ;\left(^{* *}\right) 2$ participantes não responderam em 2018/2019.

Fonte: Buriol D, et al., 2021.

Quanto aos fatores de risco, ressalta-se as diferenças entre os dois grupos. Abaixo, encontram-se dados referentes ao perfil dos fatores de risco em relação a Hipertensão Arterial Sistêmica (HAS), obesidade e doença renal, além de complicações clínicas associadas ao diabetes (Tabela 2). 
Tabela 2 - Fatores de risco e complicações clínicas e crônicas associadas ao diabetes dos pacientes atendidos em uma unidade ambulatorial.

\begin{tabular}{|c|c|c|c|c|c|}
\hline \multirow{2}{*}{ Variáveis } & \multicolumn{2}{|c|}{2016} & \multicolumn{2}{|c|}{ 2018/2019 } & \multirow{2}{*}{$\mathbf{p}^{\star}$} \\
\hline & $\mathbf{n}$ & $\%$ & $\mathbf{n}$ & $\%$ & \\
\hline \multicolumn{6}{|l|}{ Fatores de risco } \\
\hline Somente HAS & 57 & 53,8 & 19 & 22,4 & \multirow{8}{*}{0,001} \\
\hline Somente obesidade & 2 & 1,9 & 10 & 11,8 & \\
\hline Somente doença renal & 2 & 1,9 & 2 & 2,4 & \\
\hline HAS e obesidade & 13 & 12,3 & 27 & 31,8 & \\
\hline HAS e doença renal & 13 & 12,3 & 8 & 9,4 & \\
\hline HAS, obesidade e doença renal & 7 & 6,6 & 5 & 5,9 & \\
\hline Doença renal e HAS & 0 & 0 & 1 & 1,2 & \\
\hline Nenhum & 12 & 11,3 & 13 & 15,3 & \\
\hline \multicolumn{6}{|l|}{ Amputação prévia } \\
\hline $\operatorname{Sim}$ & 20 & 18,9 & 53 & 62,4 & \multirow{2}{*}{0,001} \\
\hline Não & 86 & 81,1 & 32 & 37,6 & \\
\hline \multicolumn{6}{|l|}{ Edema } \\
\hline $\operatorname{Sim}$ & 55 & 51,9 & 43 & 50,6 & \multirow{2}{*}{0,858} \\
\hline Não & 51 & 48,1 & 42 & 49,4 & \\
\hline \multicolumn{6}{|l|}{ Dormência } \\
\hline Sim & 67 & 63,2 & 52 & 61,2 & \multirow{2}{*}{0,773} \\
\hline Não & 39 & 36,8 & 33 & 38,8 & \\
\hline \multicolumn{6}{|l|}{ Formigamento } \\
\hline $\operatorname{Sim}$ & 69 & 65,1 & 49 & 57,6 & \multirow{2}{*}{0,293} \\
\hline Não & 37 & 34,8 & 36 & 42,4 & \\
\hline \multicolumn{6}{|l|}{ Dor em repouso } \\
\hline Sim & 61 & 57,5 & 42 & 49,4 & \multirow{2}{*}{0,262} \\
\hline Não & 45 & 42,5 & 43 & 50,6 & \\
\hline
\end{tabular}

Legenda: $\left(^{*}\right)$ nível de significância $p \leq 0,05$.

Fonte: Buriol D, et al., 2021.

Ainda em relação aos fatores de risco, destacou-se que sua proporção foi a mesma entre os dois grupos. Referiram ter algum fator de risco, 94 (88,7\%) pacientes da primeira amostra e 72 (84,7\%) pacientes da segunda. No grupo referente aos anos de 2018/2019, a amputação prévia em membros inferiores mostrouse presente em 53 participantes, dos quais $38(71,69 \%)$ eram do sexo masculino. Já em relação ao grupo de 2016, dos 20 pacientes que possuíam amputação prévia, 12 (60\%) eram do sexo masculino.

Quanto aos hábitos de vida, no que se refere ao tabagismo, não foi obtida significância no perfil em relação às variáveis tabagista e ex-tabagista. O perfil em relação aos hábitos de vida dos participantes apresenta-se na Tabela 3. 
Tabela 3 - Hábitos de vida dos pacientes atendidos em uma unidade ambulatorial.

\begin{tabular}{cccccc}
\hline \multirow{2}{*}{ Variáveis } & \multicolumn{2}{c}{$\mathbf{2 0 1 6}$} & $\mathbf{2 0 1 8 / 2 0 1 9}$ & \multirow{2}{*}{$\mathbf{p}^{*}$} \\
& $\mathbf{n}$ & $\%$ & $\mathbf{n}$ & $\%$ & \\
\hline Tabagismo & & & & & \\
\hline Tabagista & 11 & 10,4 & 5 & 5,9 & \\
Não tabagistas & 59 & 55,7 & 52 & 61,2 & 0,497 \\
Ex-tabagista & 36 & 34 & 28 & 32,9 & \\
\hline Atividade física & & & & & \\
\hline Sim & 31 & 29,2 & 26 & 30,6 & \multirow{2}{*}{0,840} \\
Não & 75 & 70,8 & 59 & 69,4 & \\
\hline Bebida alcoólica & & & & & \\
\hline Sim & 15 & 14,2 & 9 & 10,6 & \multirow{2}{*}{0,460} \\
Não & 91 & 85,8 & 76 & 89,4 & \\
\hline
\end{tabular}

Legenda: ${ }^{*}$ ) nível de significância $p \leq 0,05$.

Fonte: Buriol D, et al., 2021.

Com relação aos cuidados com os pés, de modo geral, notou-se a presença de práticas adequadas em ambos os grupos, como hidratar os pés e fazer o uso de meias em sapatos fechados. Contudo, foram encontradas divergências quanto ao tipo de calçado utilizado e a presença de alterações nas unhas (Tabela 4).

Tabela 4 - Cuidados com os pés dos pacientes em acompanhamento ambulatorial.

\begin{tabular}{|c|c|c|c|c|c|}
\hline \multirow{2}{*}{ Variáveis } & \multicolumn{2}{|c|}{2016} & \multicolumn{2}{|c|}{ 2018/2019 } & \multirow{2}{*}{$\mathbf{p}^{*}$} \\
\hline & $\mathbf{n}$ & $\%$ & $\mathbf{n}$ & $\%$ & \\
\hline \multicolumn{6}{|c|}{ Hidratante ou óleo nos pés ${ }^{\star *}$} \\
\hline Sim & 59 & 55,7 & 58 & 68,2 & \multirow{2}{*}{0,039} \\
\hline Não & 47 & 44,3 & 25 & 29,4 & \\
\hline \multicolumn{6}{|c|}{ Alterações nas unhas ${ }^{* \star *}$} \\
\hline Sim & 39 & 36,8 & 52 & 61,2 & \multirow{2}{*}{0,001} \\
\hline Não & 67 & 63,2 & 30 & 35,3 & \\
\hline \multicolumn{6}{|c|}{ Meias em sapatos fechados ${ }^{* *}$} \\
\hline Sim & 100 & 94,3 & 64 & 75,3 & \multirow{2}{*}{0,001} \\
\hline Não & 6 & 5,7 & 19 & 22,4 & \\
\hline \multicolumn{6}{|l|}{ Tipo de calçado** } \\
\hline Aberto & 20 & 18,9 & 48 & 56,5 & \multirow{4}{*}{0,001} \\
\hline Fechado e apertado & 1 & 0,9 & 4 & 4,7 & \\
\hline Fechado e macio & 84 & 79,2 & 29 & 34,1 & \\
\hline Sapatilha & 1 & 0,9 & 2 & 2,4 & \\
\hline
\end{tabular}

Legenda: $\left({ }^{*}\right)$ nível de significância $p \leq 0,05 ;\left(^{* *}\right) 2$ pacientes apresentaram amputação total dos pés em 2018/2019; $\left.{ }^{* * *}\right) 2$ pacientes apresentaram amputação total dos pés e 1 não respondeu em 2018/2019.

Fonte: Buriol D, et al., 2021. 
Ainda, em relação aos cuidados com os pés, do grupo de 106 pacientes, $94(88,7 \%)$ não tinham o hábito de andar sem calçados, $84(79,2 \%)$ não apresentavam rachaduras dos pés, $90(84,9 \%)$ não apresentavam calosidades e $75(70,8 \%)$ cortavam as unhas de forma inadequada, isto é, com formato arredondado. Tais frequências também foram observadas no grupo de 85 pacientes, em que 75 (90,36\%) não tinham o hábito de andar sem calçados, 63 (76,83\%) não apresentavam rachaduras dos pés, 60 (73,17\%) não possuíam calos e $58(72,50 \%)$ cortavam as unhas com formato arredondado. Não foi encontrado significância estatística em nenhuma dessas variáveis.

Quanto à orientação dos participantes entrevistados, a maioria referiu já ter recebido orientação de profissionais, sendo 90 pacientes em 2016 e 74 em 2018/2019. Ressalta-se que o enfermeiro participou das orientações de 37 (41,11\%) pacientes e de 50 (67,57\%) pacientes, respectivamente, na primeira e segunda amostra, sendo que a seguir encontra-se a distribuição dos pacientes que receberam orientação conforme seus respectivos grupos (Gráfico 1).

Gráfico 1 - Distribuição dos pacientes com DM tipo 2 em acompanhamento ambulatorial referente a orientação dos profissionais de saúde.
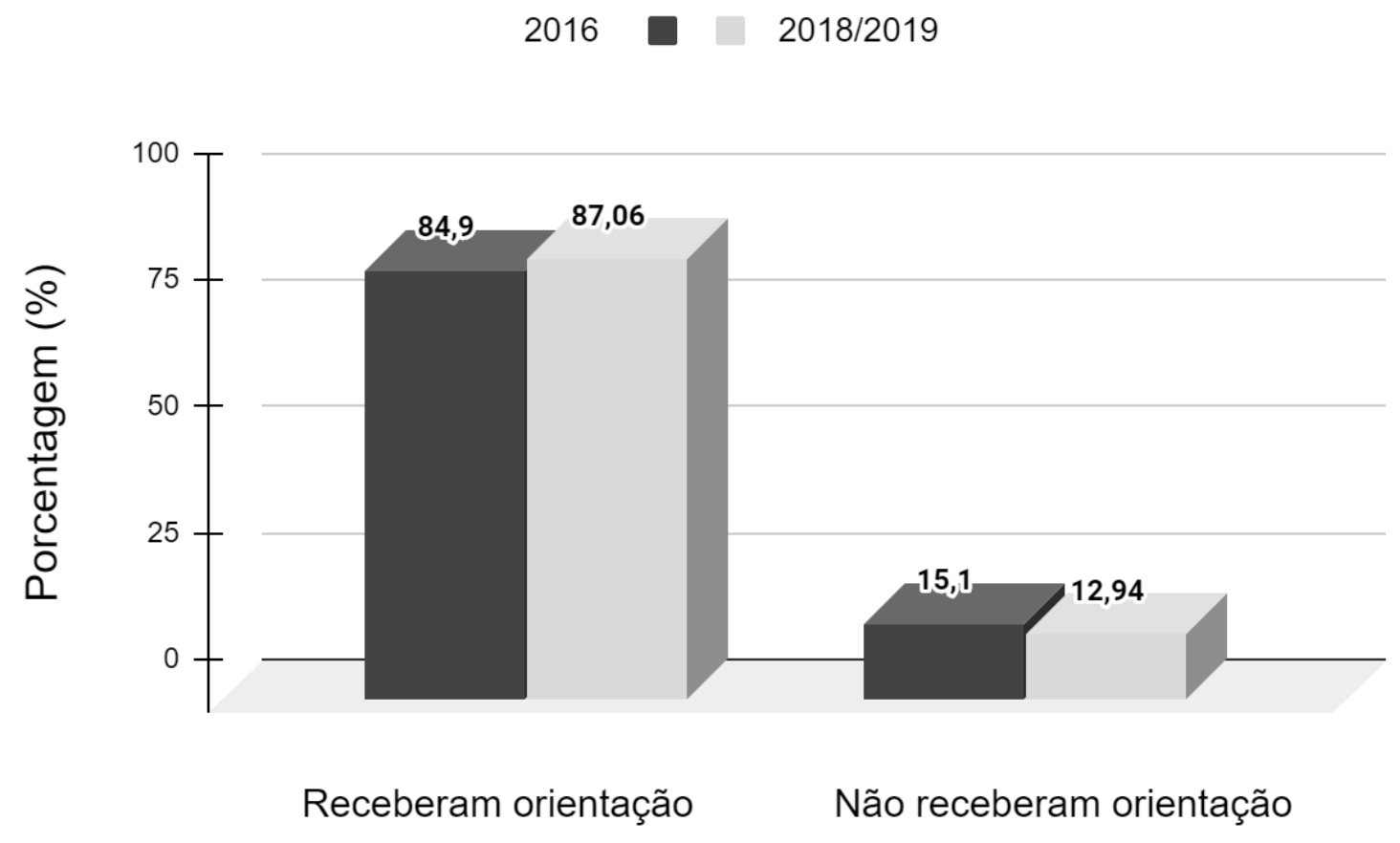

Fonte: Buriol D, et al., 2021.

\section{DISCUSSÃO}

As características sociodemográficas dos participantes da primeira amostra, atendidos por meio da consulta médica, apontam a predominância do sexo feminino, entre a faixa etária de $60-69$ anos, baixa escolaridade e renda equivalente a 1 salário mínimo. Contudo, os participantes da segunda amostra, atendidos pela equipe de enfermagem, apontam uma maior predominância do sexo masculino.

Acredita-se que o resultado observado na primeira amostra confirma a maior participação das mulheres nos serviços de saúde, o que se deve a um fato histórico (LOPES JR, et al., 2020). O processo de cuidado, nesses ambientes, possui um caráter preventivo e educativo centrados na saúde da mulher e da criança e pouco voltados para a saúde do homem, o que acaba por não incentivar o público masculino a frequentar esse ambiente (ARRUDA GO, et al., 2017). 
É importante validar, que há uma maior procura dos homens por serviços de saúde em situações extremas, em casos quando a doença evolui para um estágio mais avançado (ARRUDA GO, et al., 2017). Por conta disso, entende-se que a predominância de homens no atendimento ambulatorial, observada na segunda amostra, está relacionada à prestação de cuidados ofertados pela equipe de enfermagem a indivíduos que já possuem complicações em membros inferiores, como amputações e ulcerações prévias (LEAL TC, et al., 2020.

O pé diabético é uma das complicações mais sérias do diabetes, que pode evoluir para amputação de membros inferiores caso o controle da doença não seja feito adequadamente. A segunda amostra apontou uma maior incidência de pessoas com essa complicação, entre as quais prevaleceu o sexo masculino. $\mathrm{Na}$ primeira amostra houveram menos amputações, contudo, entre os amputados, também predominou o sexo masculino. Tais resultados corroboram com um estudo realizado em Portugal (PEDRAS S, et al., 2016). Fatores como idade avançada, baixa escolaridade e baixo status socioeconômico associados a busca tardia por serviços de saúde contribuem para o surgimento de úlceras e consequentemente levam a amputações de membros inferiores (SILVA JMTS, et al., 2017; PEDRAS S, et al., 2016).

Nas duas amostras, a faixa etária predominante se manteve entre 60 e 69 anos, o que condiz com a realidade populacional de que um quarto das pessoas com mais de 65 anos possuem diabetes (AMERICAN DIABETES ASSOCIATION, 2019). Os idosos diabéticos que se encontram nessa faixa de risco estão mais propensos ao desenvolvimento de síndromes geriátricas como polifarmácia, diminuição da capacidade cognitiva, incontinência urinária, dor persistente e maior ocorrência de quedas. $O$ impacto que essas condições trazem para a vida dessas pessoas é mais elevado e implica diretamente na capacidade da realização do autocuidado (AMERICAN DIABETES ASSOCIATION, 2019; SOCIEDADE BRASILEIRA DE DIABETES, 2019b).

Ademais, é importante salientar a respeito da renda familiar mensal ser predominantemente igual ou inferior a um salário mínimo nas duas amostras. Resultados semelhantes foram observados em um estudo também realizado em um município do Rio Grande do Sul (BRAGA DC, et al., 2015). Neste estudo, fatores socioeconômicos como baixa escolaridade e baixo nível socioeconômico associados à idade igual ou superior a 60 anos configuraram uma maior prevalência no risco de ulceração nos pés. O baixo status socioeconômico associado a baixa escolaridade e consequentemente a carência de conhecimento em saúde implicam no manejo do tratamento de pacientes com diabetes que já possuem a (SPD) Síndrome do Pé Diabético.

Nesse estudo, a maioria dos participantes vivenciavam o processo de envelhecimento, o qual é marcado pela ocorrência de diversas mudanças cruciais ao organismo, como o aumento da pressão arterial, por exemplo. Em vista disso, a presença de comorbidades associadas ao DM são bastante comuns nesse grupo etário, visto que indivíduos já acometidos por uma dessas doenças estão propensos a desenvolver outras subsequentes (SOCIEDADE BRASILEIRA DE DIABETES, 2019a).

As características clínicas do estudo ressaltaram que das condições crônicas e complicações associadas ao diabetes, a Hipertensão Arterial Sistólica (HAS) esteve presente nas duas amostras. Contudo, na segunda amostra, concomitante a HAS, também se mostrou presente a obesidade.

Nas duas amostras, os pacientes entrevistados, em sua maioria, não se declararam etilistas e tabagistas, entretanto muitos referiram ser ex-tabagistas. O consumo de álcool e tabaco por pacientes com DM tipo 2 aumenta o risco do desenvolvimento de doenças cardíacas, complicações microvasculares e dificuldades com controle glicêmico (SOCIEDADE BRASILEIRA DE DIABETES, 2019a; AMERICAN DIABETES ASSOCIATION, 2019). A prática do exercício físico, quando executada adequadamente por indivíduos com DM pode proporcionar grandes benefícios, incluindo a redução do risco cardiovascular, perda de peso e maior manejo metabólico (AMERICAN DIABETES ASSOCIATION, 2019). Entretanto, um número considerável de pacientes referiu não praticá-la. É possível que, a presença prévia de amputações tenha se tornado um fator limitador para a realização dessas atividades.

No que concerne às características de formigamento, dormência, edema e dor em repouso, estudos relataram a ocorrência desses sintomas principalmente em membros inferiores (SILVA PL, et al., 2015). A 
duração do DM constitui importante fator de risco para esse agravo, o que causa a neuropatia diabética e pode evoluir para complicações mais graves, como a amputação prévia de membros, por exemplo (SILVA PL, et al., 2015; SENTEIO JS, et al., 2018).

Em relação aos cuidados com os pés, a maior parte dos entrevistados mantinham condutas adequadas no seu dia-a-dia, as quais incluem realizar a hidratação dos pés e usar meias em sapatos fechados. Resultados semelhantes foram encontrados em outros estudos (AMERICAN DIABETES ASSOCIATION, 2019; SOCIEDADE BRASILEIRA DE DIABETES, 2019b). Na presença do DM, os pés frequentemente encontram-se com a pele ressecada, o que predispõe ao surgimento de rachaduras e ulcerações. Por esse motivo, a hidratação de membros inferiores torna-se um hábito indispensável para a pessoa que convive com DM. Entretanto, deve ser cuidado o uso de hidratante nos espaços interdigitais, a fim de evitar o aparecimento de micoses (CARLESSO GP, et al., 2017; SENTEIO JS, et al., 2018; BRASIL, 2016).

Em pessoas com diabetes que apresentam dormência em membros inferiores, o hábito de andar descalço e não utilizar calçados apropriados no dia-a-dia são causas primordiais para futuros traumas e amputações (SOCIEDADE BRASILEIRA DE DIABETES, 2019a; INTERNATIONAL WORKING GROUP ON THE DIABETIC FOOT, 2019; SENTEIO JS, et al., 2018). Na primeira amostra, o uso de calçados fechados e macios foi significativamente maior entre os pacientes. Contudo, os pacientes da segunda amostra relataram que cotidianamente optam por calçados abertos, não necessariamente adequados para sua condição clínica. Como forma de prevenção de ulcerações recomenda-se que pessoas com diabetes mellitus evitem andar sem calçados, usar sapatos sem meias ou com chinelos de sola fina e optem por sapatos confortáveis e ajustados conforme o formato dos pés (SOCIEDADE BRASILEIRA DE DIABETES, 2019a).

Na segunda amostra do estudo, foi observado um aumento no número de pacientes com alterações nas unhas, em relação à primeira. Essa condição está associada, principalmente, a infecções causadas por fungos como a onicomicose, por exemplo (SILVA JMTS, et al., 2017). Frente a essas condições ressalta-se a importância do profissional de saúde no manejo do cuidado com os pés dos pacientes. Após fazer uma inspeção adequada é válido incentivá-los no autocuidado, realizar a inspeção diária dos pés, manter uma higiene adequada e utilizar calçados adequados para prevenir infecções e traumas teciduais (SILVA JMTS, et al., 2017; SENTEIO JS, et al., 2018).

A maioria dos entrevistados com DM tipo 2 foram orientados por profissionais de saúde. Esse dado pode ter contribuído para a realização dos devidos cuidados em relação aos pés de um elevado número de pacientes, conforme pode ser evidenciado em outro estudo (REZENDE NETA DS, et al., 2015).

Destarte, foi possível observar, ainda, a relação estatisticamente significativa das orientações disponibilizadas pelos profissionais de saúde quanto à aderência às atividades de autocuidado com os pés e ao controle do tratamento da doença. Diante disso, destaca-se o papel do enfermeiro enquanto responsável pela adesão do paciente ao tratamento e estímulo à incorporação de práticas adequadas para o controle do DM, sobretudo, o cuidado com os pés (SILVA PL, et al., 2015; REZENDE NETA DS, et al., 2015).

\section{CONCLUSÃO}

Destaca-se a prevalência de pacientes do sexo feminino na primeira amostra e de pacientes do sexo masculino na segunda amostra, bem como a ocorrência da presença de amputação prévia como complicação associada ao DM mais prevalente entre os entrevistados do sexo masculino, principalmente na segunda amostra. Espera-se que o conhecimento dessas características possa contribuir para o desenvolvimento de práticas de educação em saúde e de estratégias de prevenção para o surgimento de maiores agravos desta população, de modo a intensificar as ações de promoção de saúde na rotina cotidiana dos profissionais e promover o tratamento mais adequado para os pacientes. Dessa forma, ressalta-se que o enfermeiro tem recebido um importante papel no processo de educação e cuidado dos pacientes com DM. Portanto, o mesmo deverá utilizar o seu conhecimento para proporcionar as orientações e ações necessárias, contribuindo para a promoção de saúde, prevenção do pé diabético, estimulação do autocuidado, melhora da qualidade de vida e auxílio do convívio dos pacientes com a condição crônica. 


\section{REFERÊNCIAS}

1. AMERICAN DIABETES ASSOCIATION. Standards of medical care in diabetes - 2019. Lifestyle Management. United States. Diabetes Care 2019; 42 (Suppl. 1): S51.

2. ARRUDA GO, et al. Prevalência e fatores associados à utilização de serviços públicos de saúde por homens adultos. Ciênc. Saúde Colet, 2017; 22(1):279-290.

3. BRAGA DC, et al. Evaluation of neuropathy and vascular complications in patients with diabetes mellitus in a rural town of Santa Catarina. Rev. AMRIGS, 2015; 59 (2): 78-83.

4. BRASIL. Resolução número 466: de 12 de dezembro de 2012. Dispõe sobre as diretrizes e as normas regulamentadoras de pesquisa envolvendo seres humanos. Brasília (DF): Ministério da Saúde; 2012 [Internet]. Disponível em: http://bvsms.saude.gov.br/bvs/saudelegis/cns/2013/res0466_12_12_2012.html. Acessado em: 14 de fevereiro de 2021.

5. BRASIL. Ministério da Saúde. Secretaria de Ciência, Tecnologia e Insumos Estratégicos, Departamento de Ciência e Tecnologia. Agenda nacional de prioridades de pesquisa em saúde. 2 ed. 4. reimp. Brasília (DF): Editora do Ministério da Saúde; $2015 \quad$ [Internet]. Disponível em: http://bvsms.saude.gov.br/bvs/ publicacoes/agenda_nacional_prioridades_2ed_4imp.pdf. Acessado em 06 de fevereiro de 2021.

6. BRASIL. Ministério da Saúde. Secretaria de Atenção à Saúde, Departamento de Atenção Básica. Manual do pé diabético: estratégias para o cuidado da pessoa com doença crônica. Brasília (DF): Ministério da Saúde; 2016. Disponível em: http://www.as.saude.ms.gov.br/wp-content/uploads/2016/06/manual_do_pe_diabetico. pdf. Acessado em 27 de março de 2021.

7. CARLESSO GP, et al. Avaliação do conhecimento de pacientes diabéticos sobre medidas preventivas do pé diabético em Maringá (PR). J. vasc. bras, 2017; 16(2): 113-118.

8. INTERNATIONAL DIABETES FEDERATION. IDF Diabetes Atlas. 9. ed. Brussels, Belgium: IDF; 2019,176 p.

9. INTERNATIONAL WORKING GROUP ON THE DIABETIC FOOT. Practical guidelines on the prevention and manegement of diabetc foot disease. IWGDF Pratical Guidelines; 2019, 194 p.

10. LEAL TC, et al. Knowledge and practices of people with type 2 diabetes mellitus about diabetic foot. Research, Society and Development, 2020; 9(7): 1-15, e618974511.

11. LOPES JR, et al. Perfil epidemiológico de usuários atendidos em ação de saúde na baixada litorânea do Rio de Janeiro. Rev Fun Care Online, 2020; 12:258-263.

12. NETO MO, et al. Evaluation of self-care for diabetic foot prevention and clinical examination of the feet in a diabetes mellitus reference center. J. Health Biol. Sci, 2017; 5(3):265-271.

13. PEDRAS S, et al. Sociodemographic and clinical characteristics of patients with diabetic foot ulcer. Rev. Assoc. Med. Bras, 2016; 62(2): 171-178.

14. REZENDE NETA DS, et al. Adesão das pessoas com diabetes mellitus ao autocuidado com os pés. Rev Bras Enferm, $2015 ; 68(1): 111-6$.

15. SENTEIO JS, et al. Prevalência de fatores de risco para o desenvolvimento de pé diabético. Rev Fun Care Online, 2018; 10(4):919-925.

16. SILVA JMTS, et al. Fatores associados à ulceração nos pés de pessoas com diabetes mellitus residentes em área rural. Rev. Gaúcha Enferm, 2017;38(3): e68767

17. SILVA PL, et al. Care of the feet: the knowledge of individuals with diabetes mellitus registered family health program. Enferm. Glob, 2015; 14(37):52-64.

18. SOCIEDADE BRASILEIRA DE DIABETES. Diretrizes da Sociedade Brasileira de Diabetes 2019-2020. São Paulo: Editora Clannad; 2019a, $491 \mathrm{p}$.

19. SOCIEDADE BRASILEIRA DE DIABETES. Posicionamento Oficial SBD n. 07/2019 Abordagem da pessoa idosa com diabetes 2019-2020. São Paulo: Editora Europa Press; 2019b, 28 p.

20. TESTON EF, et al. Fatores de risco para ulceração no pé de indivíduos com diabetes mellitus tipo 2. Cogitare Enferm, $2017 ; 22(4): 1-14$. 